\title{
MANAGEMENT OF THE FRACTURED SCAPHOID USING A NEW BONE SCREW
}

\author{
TIMOTHY J. HERBERT, WILLIAM E. FISHER
}

From the Sydney Hospital and the University of New South Wales, Sydney

\begin{abstract}
A new and simple operative technique has been developed to provide rigid internal fixation for all types of fractures of the scaphoid. This involves the use of a double-threaded bone screw which provides such good fixation that, after operation, a plaster cast is rarely required and most patients are able to return to work within a few weeks. A classification of scaphoid fractures is proposed.

The indications for operation included not only acute unstable fractures, but also fractures with delayed healing and those with established non-union; screw fixation was combined with bone grafting to treat nonunion. In a prospective trial, 158 operations using this technique were carried out between 1977 and 1981. The rate of union was 100 per cent for acute fractures and 83 per cent overall. This method of treatment appears to offer significant advantages over conventional techniques in the management of the fractured scaphoid.
\end{abstract}

Fracture of the scaphoid is a common condition whose management remains controversial. Conventional methods of treatment involve the use of a standard scaphoid plaster, incorporating the base of the thumb. Opinions differ widely as to the period of immobilisation required, the optimal position of the wrist and the need to include the elbow in the plaster (London 1961). Little has been written about the complications of conservative treatment; these include inability to work while in plaster, stiffness of the wrist afterwards, muscle wasting, weakness and malunion. The true incidence of non-union after conservative treatment is difficult to estimate, and most reported series do not define clearly the criteria of union or specify the length of clinical and radiographic followup (Leslie and Dickson 1981; Cooney, Dobyns and Linscheid 1980).

There is no doubt that symptomatic non-union of the scaphoid remains a common and disabling problem. Over 180 patients with non-union have presented to us for treatment during the last seven years; over half had previously undergone conservative treatment of an acute fracture. Radiographs of the scaphoid are notoriously difficult to interpret, and although the fracture may appear to be united after treatment, radiographic followup may later show the development of established nonunion (Fig. 1). Our experience suggests that the true

T. J. Herbert, FRCS, Orthopaedic Surgeon

Sydney Hospital, Sydney, NSW, Australia.

W. E. Fisher, BSc, BE

School of Mechanical and Industrial Engineering, University of New South Wales, Sydney, NSW 2033, Australia.

Requests for reprints should be sent to Mr T. J. Herbert, 135 Macquarie Street, Sydney, NSW 2000, Australia.

(C) 1984 British Editorial Society of Bone and Joint Surgery $0301-620 \mathrm{X} / 84 / 1030 \$ 2.00$ incidence of non-union after conservative treatment is of the order of 50 per cent.

The management of symptomatic non-union usually involves some form of bone-grafting operation. The most successful appears to be the Matti-Russe procedure which gives a rate of union of approximately 85 per cent (Russe 1960; Dooley 1968; Mulder 1968; Trojan 1974). However, this operation demands immobilisation of the wrist in plaster for up to six months after operation, so that stiffness is almost inevitable. It is important to recognise that the majority of patients are active young men who cannot afford to be off work for lengthy periods, and it is not surprising that a significant number refuse to undergo bone-grafting procedures.

In spite of these problems, internal fixation of the scaphoid has never been popular. There are several reasons for this: the operation is technically demanding (McLaughlin 1954); firm fixation is difficult to achieve (Gasser 1965); and the failure rate is unacceptably high (Maudsley and Chen 1972). We therefore started in 1975 to investigate alternative methods of internal fixation designed to overcome these problems. This led to the development of an entirely new type of bone screw, together with the instrumentation to facilitate its insertion.

\section{DEVELOPMENT AND DESIGN}

Study of the problems associated with internal fixation of the scaphoid suggested that the main factor was the lack of a suitable implant for fixation of small fragments of cancellous bone. The use of Kirschner wires is, perhaps, the simplest method, but it does not produce rigid fixation, so that additional immobilisation in plaster is required. The ends of the wires are left protruding from 


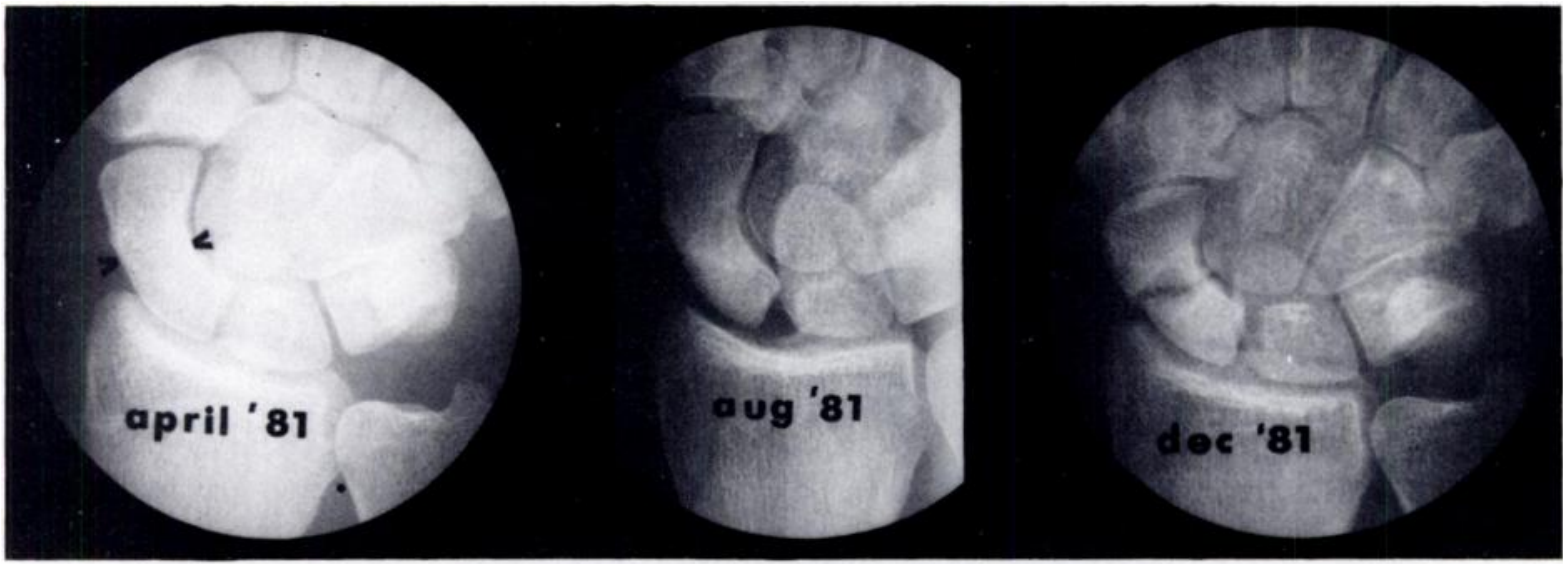

Fig. 1

Radiographs showing acute fracture of scaphoid. In August, after 14 weeks in plaster, there is apparent union, but four months later non-union is established.

the bone, often in close proximity to a joint, so that unrestricted movement is not possible. A second operation to remove the wires is usually necessary.

Since fractures of the scaphoid are intra-articular, the use of small plates or staples is inappropriate and, in any case, such devices are unlikely to give rigid internal fixation.

Clearly the ideal method of fixation must involve the use of an intramedullary implant which provides compression across the fracture. However, experience with the use of standard cancellous or lag screws has shown a number of significant problems which commonly

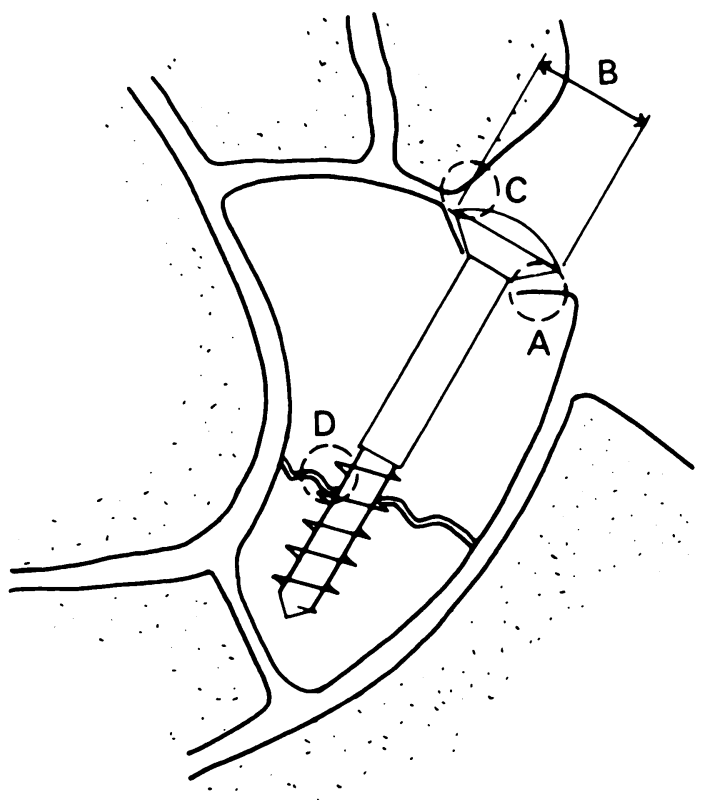

Fig. 2

Diagram showing common problems with standard screw fixation of scaphoid: A, fracture or resorption of outer cortex under concentrated loading from head of screw; B, large diameter of screw relative to bone, making accurate alignment difficult: $C$, protrusive screw head interfering with adjacent structures and precluding insertion through articular cartilage: $D$, thread crossing fracture, preventing compression. lead to failure of fixation (Fig. 2). Furthermore, insertion of the screw often presents considerable technical difficulties: the line and length may be difficult to determine and normally requires radiographic control during the operation; and the two bone fragments tend to be pushed apart as the thread of the screw crosses the fracture, leading to poor fixation. Thus, the use of some form of guiding jig is desirable, both to hold the fragments together during insertion of the screw and to align the instruments. The size of the head on a conventional bone screw has hindered the development of a suitable jig.

In order to overcome these problems an entirely new type of screw has been developed which has a second thread in place of the normal screw head (Fig. 3). This

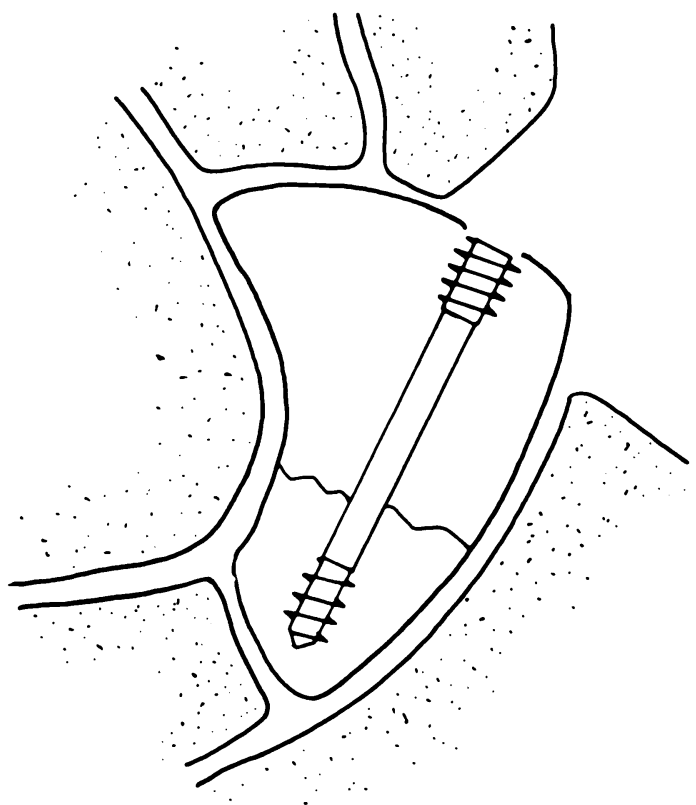

Fig. 3

Diagram showing design of the new bone screw. Note the reduced diameter, the lack of protrusive head, threads that engage both fragments, and the short leading thread for fixation of small proximal fractures. 


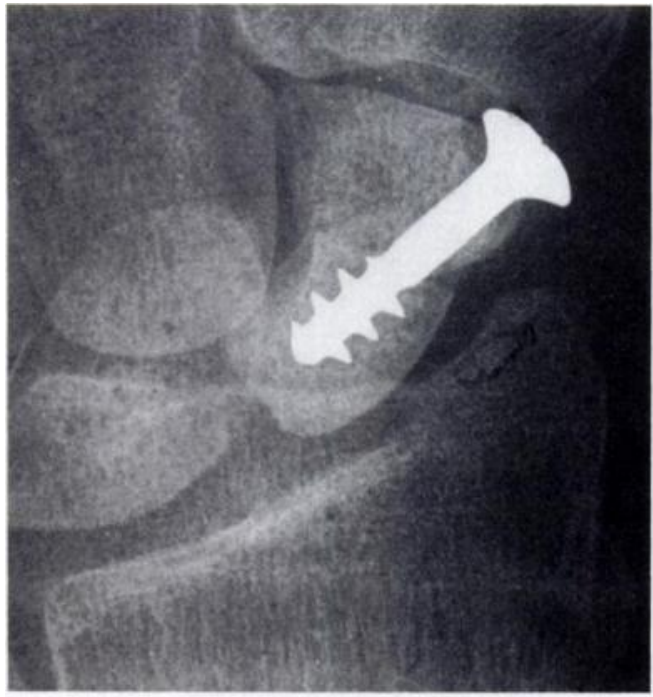

Fig. 4

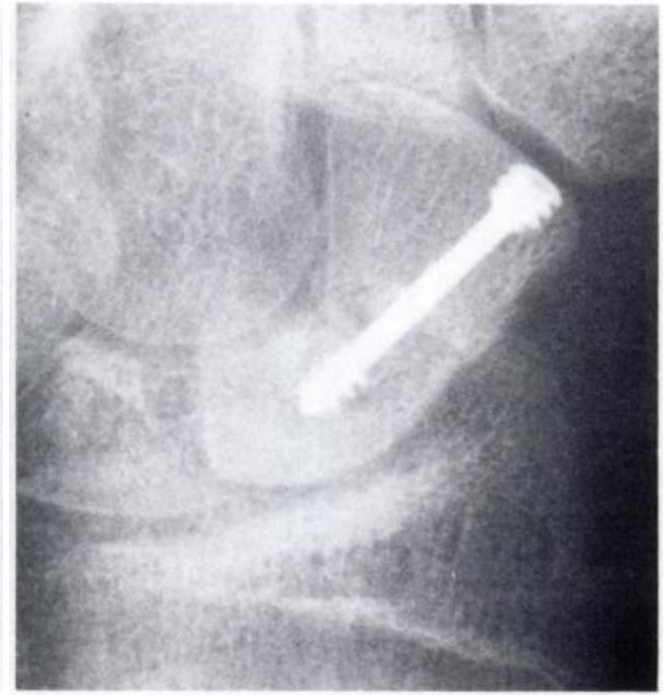

Fig. 5

Comparison with the standard screw (Fig. 4) emphasises the smaller size of the new bone screw (Fig. 5) which allows more accurate positioning. Note also the firm hold in both bone fragments given by the new screw and its insertion through the distal articular surface of the scaphoid.

design allows for a significant reduction in the overall diameter of the implant, but at the same time gives firm fixation of both fragments. The length of thread on the leading end of the screw is sufficiently short to allow firm fixation of small proximal pole fractures of the scaphoid. The absence of a protrusive head allows the screw to be inserted through articular cartilage where necessary (Figs 4 and 5).

In order to apply compression, the thread on the leading end of the screw has a greater pitch than that at the trailing end, so that fragments are drawn together as the screw is inserted (Fig. 6). The amount of compression across the fracture depends not only on the pitch differential but also on the number of turns applied to the
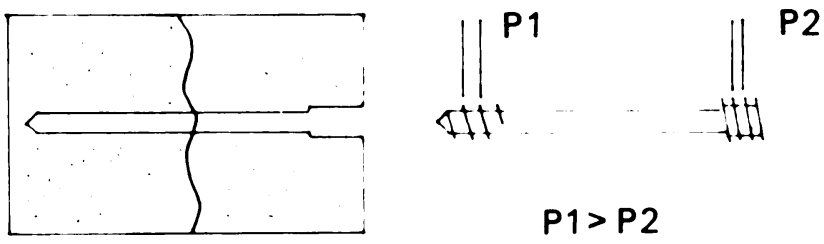

P1 $>$ P2

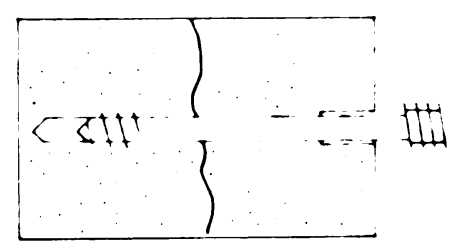

Fig. 6

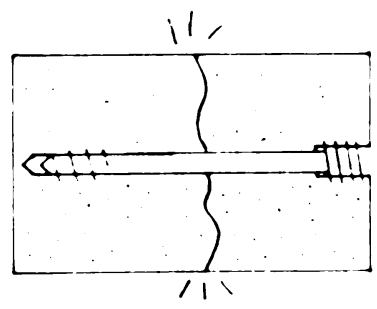

Difference in pitch between the leading thread $(\mathrm{P} 1)$ and the trailing thread (P2) governs the rate of "take up" or drawing together of the two bone fragments, producing compression screw after the trailing thread enters the bone. With the current design the two fragments must be held in apposition before insertion of the screw, and this is normally achieved by the use of a guiding jig (Fig. 7). Drilling and tapping are carried out by hand (Figs 8 to 11 ), and the screw, which has a hexagonal driving socket, is inserted through the jig; the jig is removed once the screw is fully buried.

The same technique is used in conjunction with bone grafting for non-union; after resection of the pseudarthrosis and insertion of the graft, the jig is clamped firmly into place. The screw passes through the middle of the graft, providing rigid fixation (Fig. 12).

The screws range in length from 16 millimetres to 30 millimetres and are 4 millimetres in diameter. They are manufactured from titanium alloy and appear to be inert so that later removal is not indicated.

\section{METHODS}

Operative technique. The operation is carried out through an anterior approach centred over the tubercle of the scaphoid and curved distally into the base of the thenar eminence. The flexor carpi radialis tendon is retracted towards the ulna, and the capsule of the wrist is incised longitudinally in the bed of the tendon to display the anterior surface of the scaphoid and the fracture. The distal end of the scaphoid is exposed and mobilised by a transverse incision of the capsule of the trapezioscaphoid joint. The fracture is then reduced or, in the case of nonunion, prised open to correct the flexion deformity, preserving a soft-tissue bridge posteriorly.

All fibrous tissue and sclerotic bone are carefully resected, which often leaves a large defect. In such cases, a corticocancellous bone graft, taken from the outer aspect of the iliac crest, is prepared to fill the defect. Any 


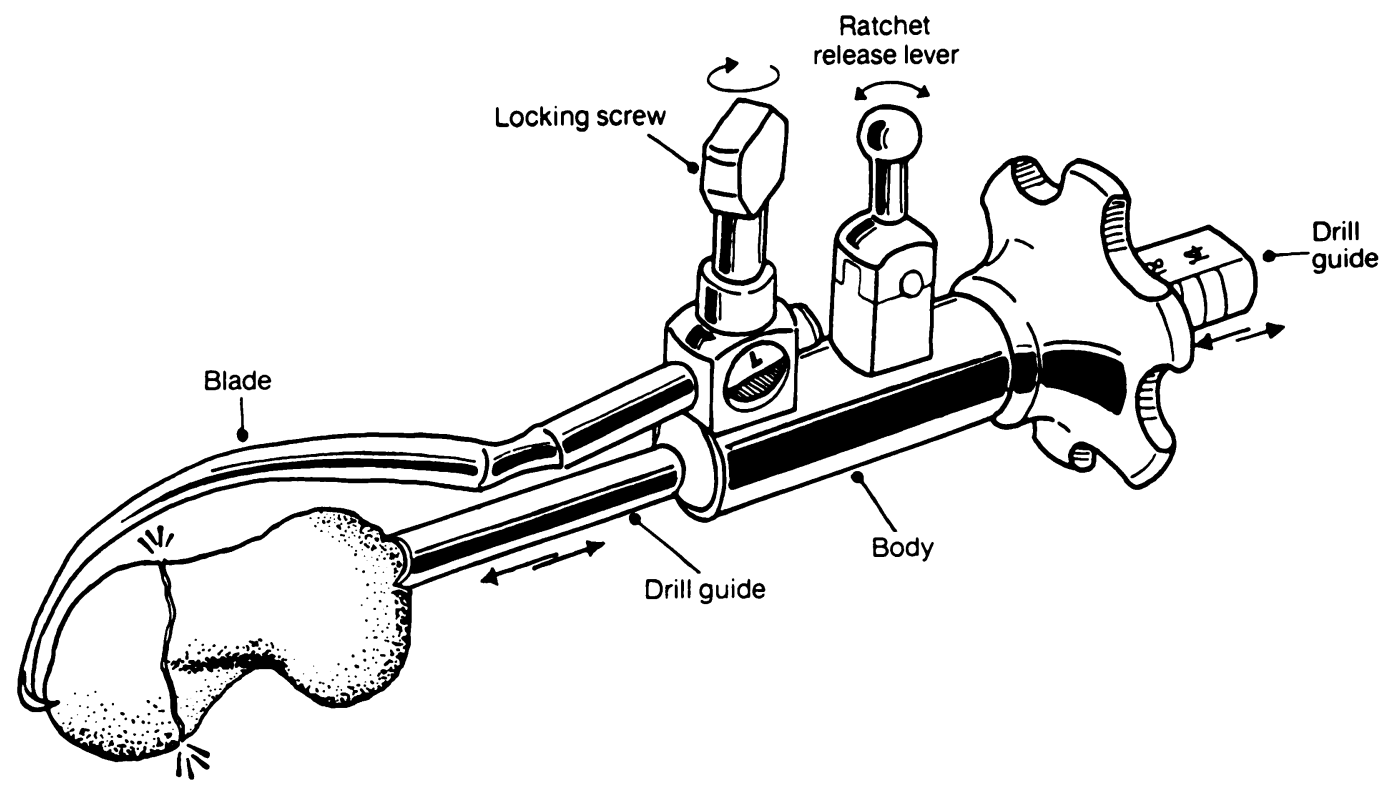

Fig. 7

Diagram showing the guiding jig. It consists of a calibrated drill guide secured by a ratchet within the main body of the jig, to which is attached a blade to engage the proximal pole of the bone. The jig is aligned under direct vision (radiographic control is not required) and the drill guide is advanced by pressure of the thumb onto the tubercle, clamping the two fragments firmly together.
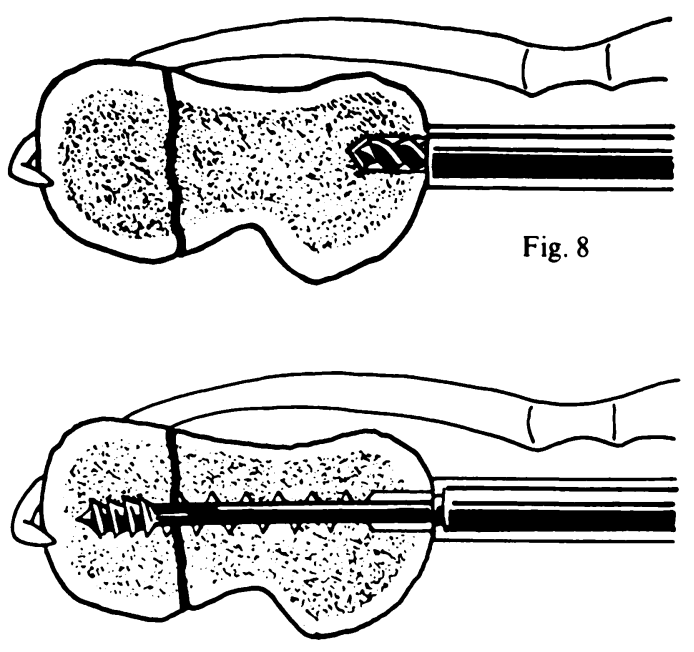

Fig. 10

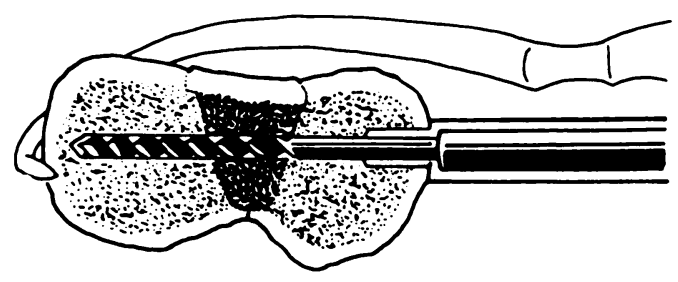

Fig. 12
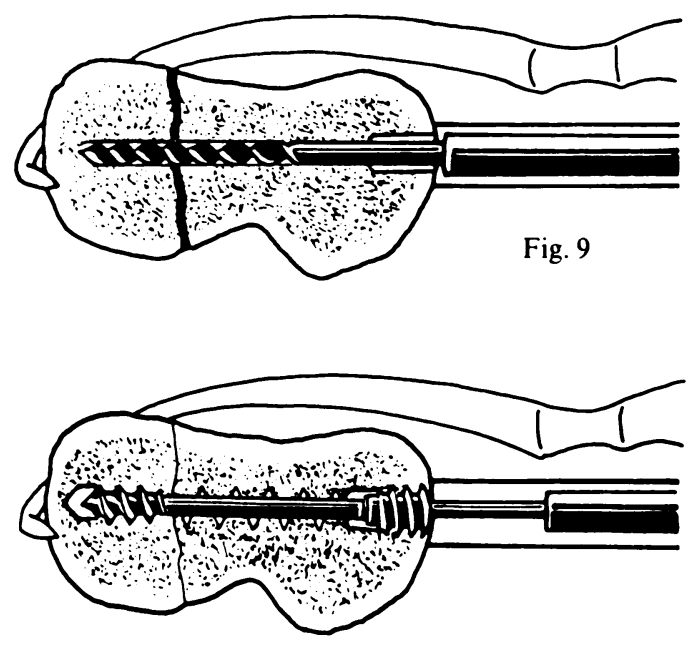

Fig. 11

Diagrams showing the stages of instrumentation. Figure 8-The pilot drill (for trailing end of screw). Figure 9Long drill (for leading end of screw). Figure 10-Tap (for leading thread). Figure $11-$ The screw is inserted through the jig (the trailing thread is self-tapping). The jig is used to align all instruments and a built-in stop ensures drilling to the exact length required.

Figure 12-Diagram showing the technique of screw fixation in conjunction with bone grafting. 
gap between the graft and the fracture-ends is packed with cancellous bone chips.

Care is taken with accurate positioning of the jig to ensure the best line for insertion of the screw (Fig. 13). The blade is hooked around the proximal pole and the jig is clamped firmly into position. The length of the screw required is read directly off the calibrated guide, the screw is fully inserted through the jig, and the jig is then removed. Extra compression may be achieved by further turns of the screw. The graft is trimmed to ensure that it is completely smooth and the stability of fixation is checked by moving the wrist in all directions. The anterior capsule of the wrist is carefully repaired using fine non-absorbable sutures, the skin closed and a firm compression bandage applied. Once the sutures have been removed, most patients are fitted with a lightweight Orthoplast splint to protect the repair from further injury during the healing period. However, removal of the splint and active mobilising exercises are encouraged from the outset and the patient is normally allowed to return to work once the wound has healed. A postoperative radiograph is taken to confirm satisfactory positioning of the screw and graft (Figs 14 and 15).

Indications. Internal fixation was considered desirable in all cases where control of instability would not only ensure a satisfactory outcome, but would also allow for early functional recovery of the wrist. Thus, in the treatment of acute fractures, it became essential to differentiate between those with a good prognosis, which would unite within six to eight weeks, and those that would not. A retrospective study of over 200 scaphoid fractures indicated that undisplaced or stable fractures had a good prognosis with conservative treatment if treated early. However, those fractures presenting late,

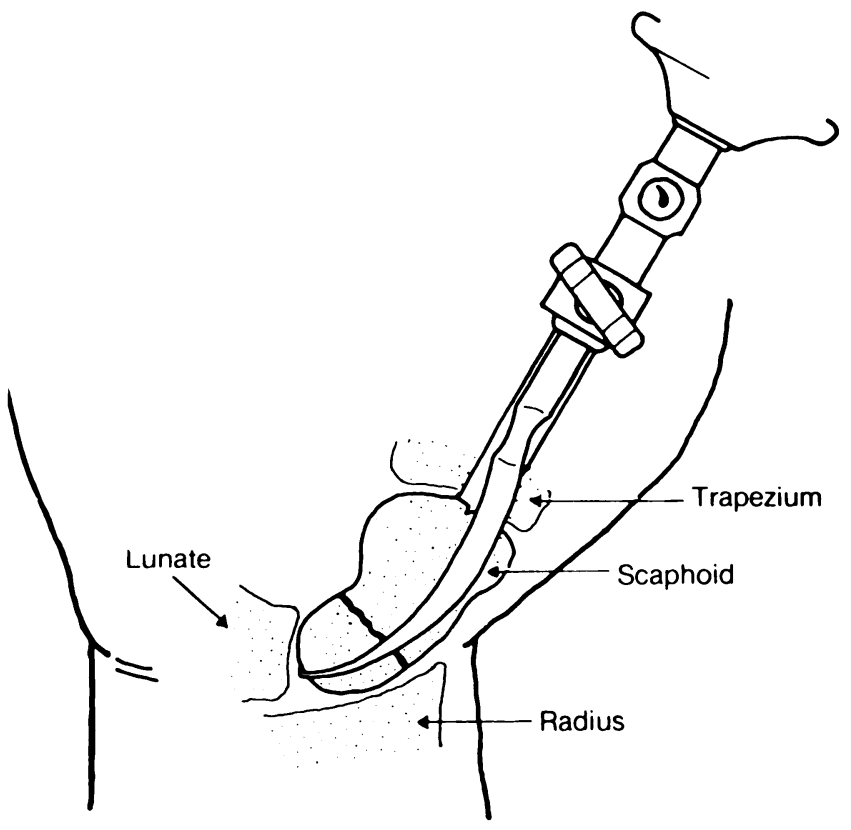

Fig. 13

Diagram showing positioning of jig on the scaphoid.

as well as those showing instability at the fracture site (of which five common patterns were recognisable), had a much poorer prognosis (Herbert 1974). Similarly, fractures showing signs of delayed union after six to eight weeks in plaster, as well as those with clear signs of nonunion, did not appear to do well with conservative treatment.

Thus, all scaphoid fractures presenting for treatment during the period of this trial were classified according to their radiographic appearance (Table I; Fig. 16). In order to recognise these different fracture types, it is essential

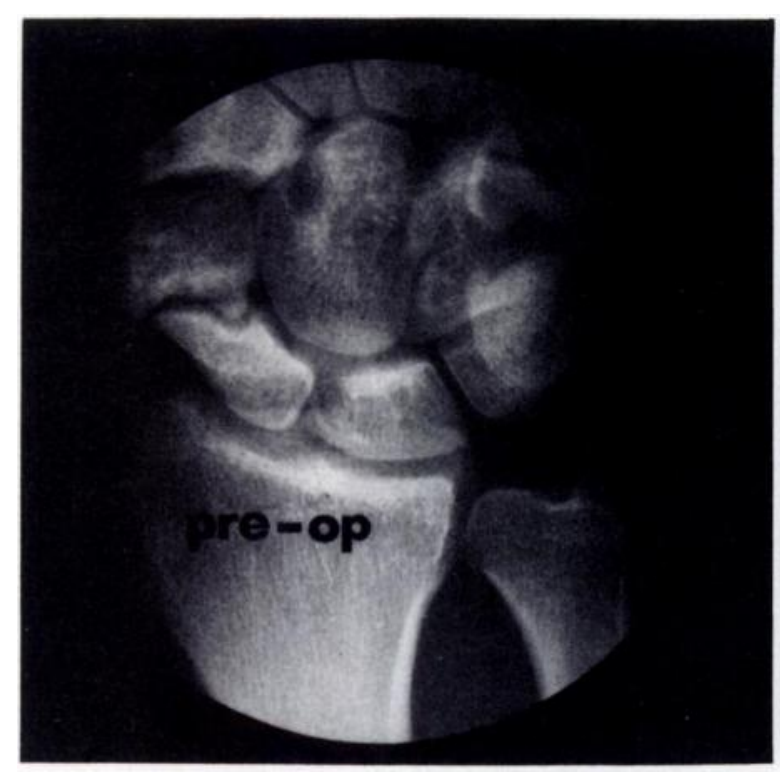

Fig. 14

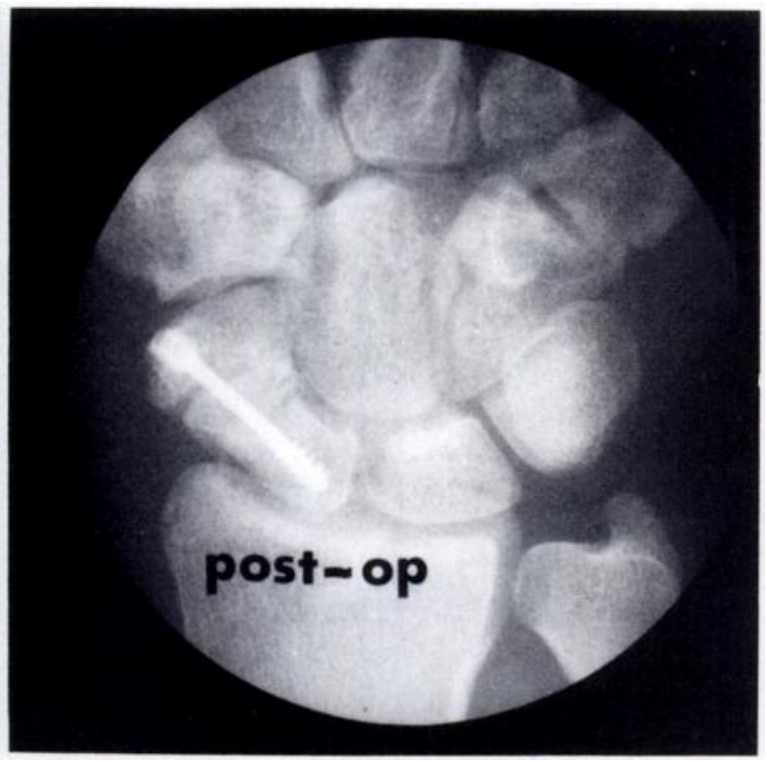

Fig. 15

Figure 14 Radiograph showing established non-union of scaphoid with deformity (sclerotic non-union-Type D2). Figure 15Radiograph taken after resection of the pseudarthrosis, bone grafting and screw fixation. Note the extent of the bone graft and firm compression. 

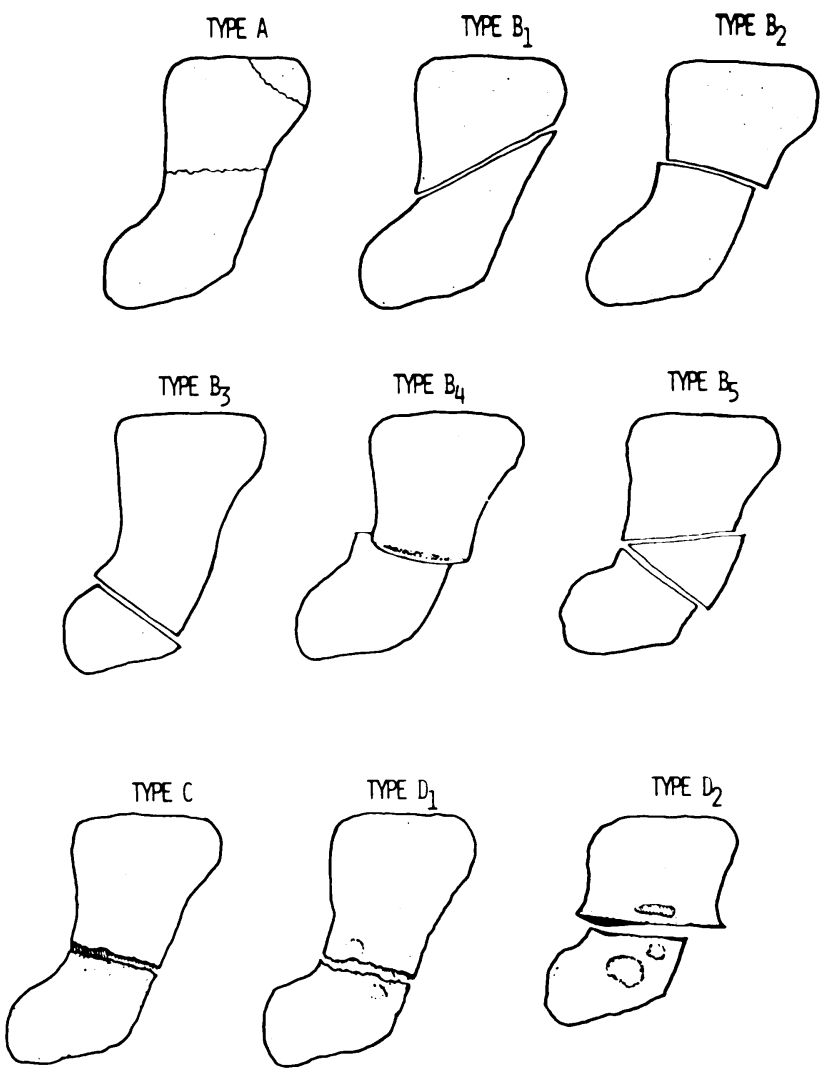

Fig. 16

Classification of scaphoid fractures according to their radiographic appearance (see also Table I).

that adequate radiographs are taken of both wrists (Figs 17 and 18). These should include posteroanterior views in full ulnar and radial deviation, as well as 45-degree obliques and true laterals with the wrist in neutral flexion. Type $A$ (acute stable) fractures were treated conservatively and have not been included in this series.

Type $B$ (acute unstable) fractures were treated by primary
Table I. Classification of scaphoid fractures (see also Fig. 16)

Type A (acute stable fractures)

A1: fractures of the tubercle

A2: undisplaced "crack" fracture of waist

Type $B$ (acute unstable fractures)

B1 : oblique fractures of distal third

B2: displaced or mobile fractures of waist

B3: proximal pole fractures

B4: fracture dislocations of carpus

B5: comminuted fractures

Type $C$ (delayed union)

Type D (established non-union)

D1: fibrous non-union

D2: sclerotic non-union (pseudarthrosis)

internal fixation as soon as practicable after the injury. Type $C$ fractures (which showed signs of delayed union after six or more weeks in plaster) were treated operatively. The plaster was removed at least two weeks before operation to mobilise the wrist and recalcify the bone. Similarly, "late" fractures presenting some weeks after injury were included in this group and were treated by primary screw fixation.

Type $D$ fractures (with established non-union) must be differentiated from acute fractures, even when there is no definite history of previous injury. In this group screw fixation combined with bone grafting is indicated if the patient has significant symptoms or is at risk of developing secondary degenerative changes in the radiocarpal joint.

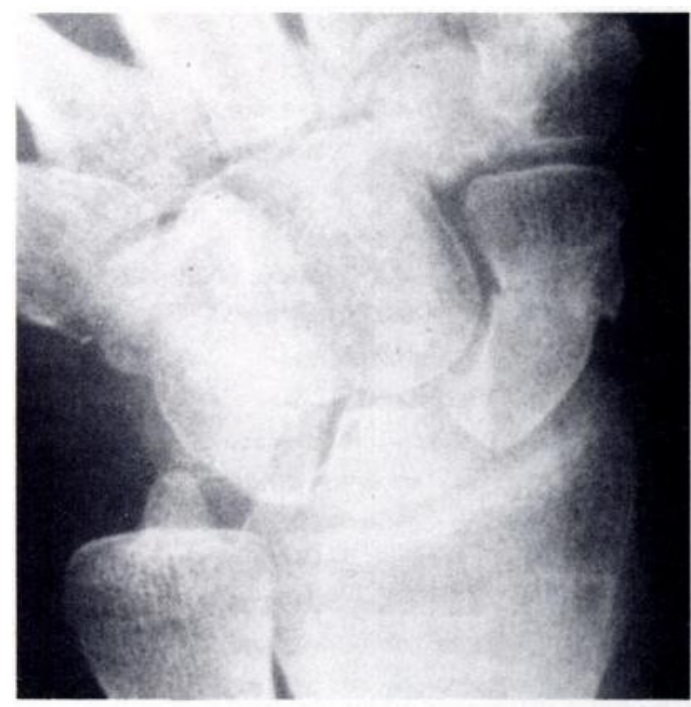

Fig. 17

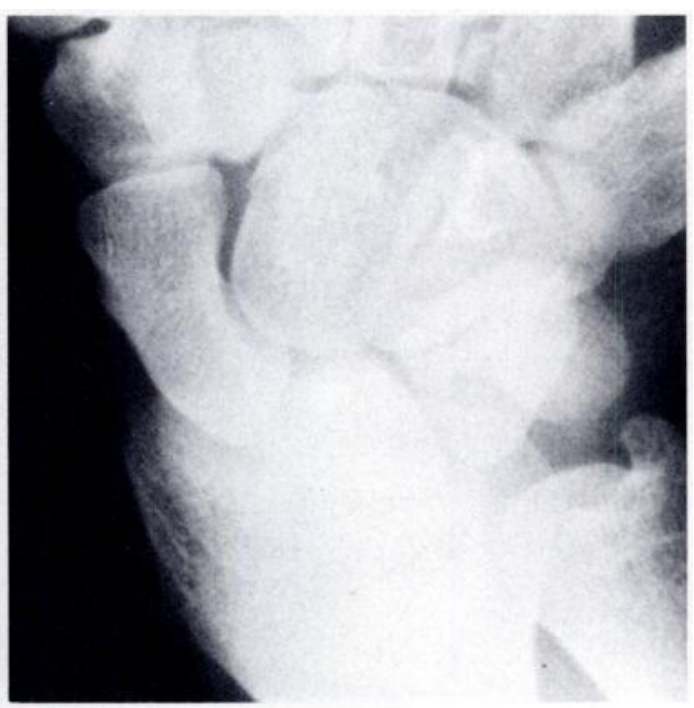

Fig. 18

Posteroanterior radiographs of both wrists in full ulnar deviation to demonstrate displacement and instability of the acute fracture of the waist of the left scaphoid (Type B2). 


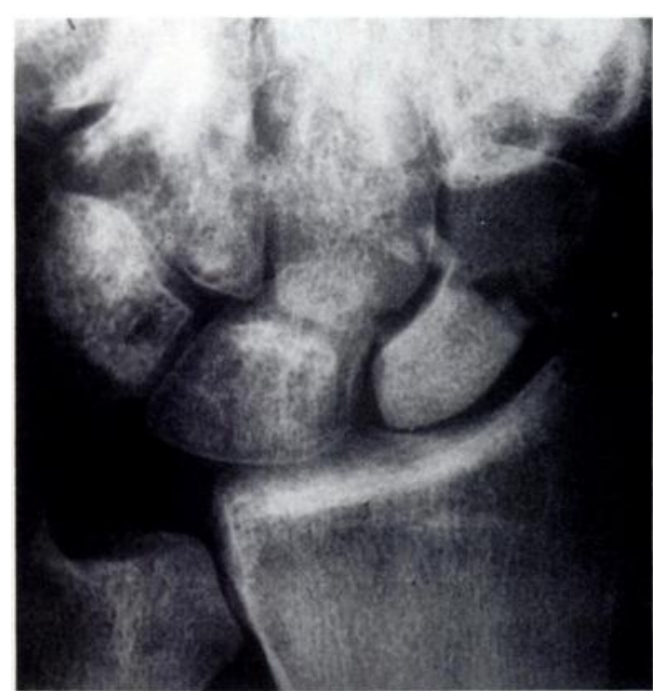

Fig. 19

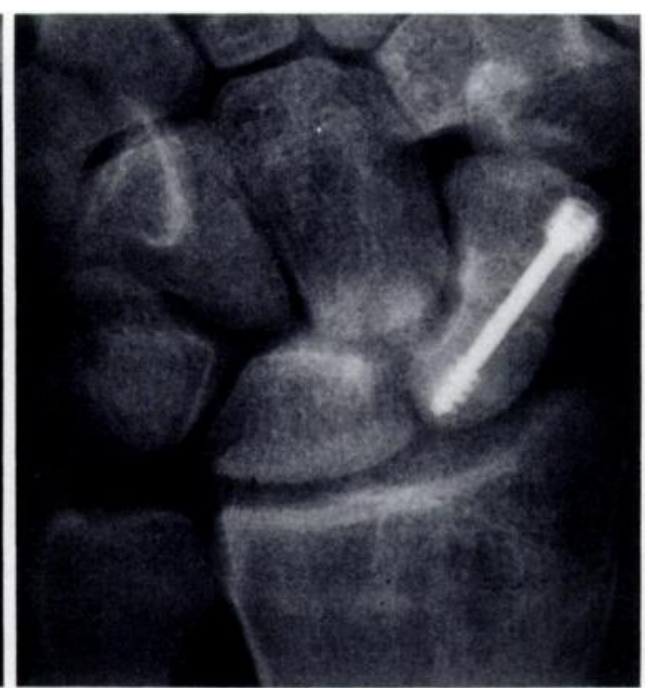

Fig. 20

Figure 19 Radiograph showing delayed union (Type C) with relative ischaemia of the proximal scaphoid fragment after treatment in plaster for eight weeks. Figure 20-Radiograph taken 12 weeks after screw fixation showing union of fracture and a return to normal density.

Contra-indications. In the case of long-standing pseudarthrosis with severe osteoarthritis, reconstruction is usually contra-indicated and an alternative procedure preferred. Relative ischaemia of the proximal scaphoid fragment, a common finding after immobilisation of the wrist, is not a contra-indication. Indeed over 50 per cent of Type $C$ fractures treated in this series showed significant radiographic evidence of ischaemia but subsequently returned to normal after screw fixation and union of the fracture (Figs 19 and 20). True avascular necrosis was found to be associated with fractures of the proximal pole with established non-union; in such cases Silastic replacement

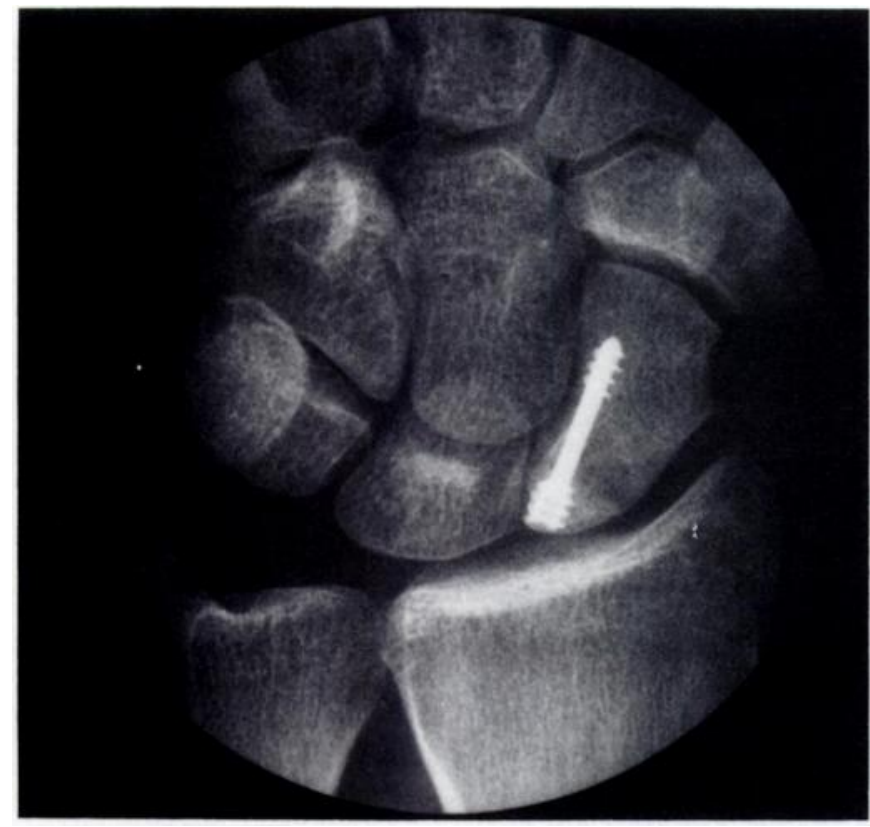

Fig. 21

Radiograph showing retrograde screw fixation combined with a cancellous bone graft for a small proximal pole fracture (Type B3). of the necrotic fragment was the treatment of choice. However in the case of extremely small proximal pole fragments, screw fixation was carried out in a retrograde manner provided that the fragment of bone appeared viable at operation (Fig. 21).

\section{MATERIAL}

One hundred and fifty-eight operations using the new bone screw were performed between June 1977 and August 1981. The average age of the patients was 24.8 years (range 16 to 43 years); 94 per cent (148 patients) were male. The dominant hand was affected in 59 per cent ( 94 patients). There were 22 Type B fractures (acute unstable), 21 Type $C$ fractures (delayed union), and 115 symptomatic non-unions of which 38 were Type D1 (fibrous non-union) and 77 Type D2 (sclerotic nonunion).

Common to all patients was a history of forced dorsiflexion of the wrist; one third of the fractures had resulted from sporting injuries, although motor cycle accidents were also a common cause. Only six patients (all women) sustained their fractures in a simple fall.

There were 15 fracture-dislocations, all associated with a severe degree of trauma. There were 54 fractures of the proximal pole which also tended to be associated with the more severe type of injury.

Assessment. Specially designed forms were used to record all information on patients included in this trial. Clinical examination included the measurement of swelling and tenderness in the "anatomical snuff-box" and the range of movements of the wrist in all four directions; the strength of hand grip was assessed using a dynamometer. These measurements were compared with the opposite normal side and used to assess overall function of the wrist.

Clinical and radiographic follow-up was carried out 
Table II. Grading of results

\begin{tabular}{|c|c|c|c|}
\hline Grade & Patient satisfaction & Clinical results & Radiographic results \\
\hline 0 & $\begin{array}{l}\text { Very happy } \\
\text { Asymptomatic }\end{array}$ & $\begin{array}{l}\text { Normal function } \\
\text { Unrestricted use }\end{array}$ & $\begin{array}{l}\text { Sound union } \\
\text { No deformity }\end{array}$ \\
\hline 1 & $\begin{array}{l}\text { Improved } \\
\text { Minimal symptoms }\end{array}$ & $\begin{array}{l}\text { Minimal loss of function } \\
\text { Unrestricted use }\end{array}$ & $\begin{array}{l}\text { Apparent union } \\
\text { Minimal deformity }\end{array}$ \\
\hline 2 & $\begin{array}{l}\text { Unchanged } \\
\text { Moderate symptoms }\end{array}$ & $\begin{array}{l}\text { Moderate loss of function } \\
\text { Some restriction }\end{array}$ & $\begin{array}{l}\text { Doubtful union } \\
\text { Marked deformity }\end{array}$ \\
\hline 3 & $\begin{array}{l}\text { Worse } \\
\text { Severe symptoms }\end{array}$ & $\begin{array}{l}\text { Marked loss of function } \\
\text { Restricted use }\end{array}$ & $\begin{array}{l}\text { Non-union } \\
\text { Loosening of screw }\end{array}$ \\
\hline
\end{tabular}

at regular intervals for a minimal period of one year (although patients in whom the outcome was clear after six months have been included in this series). The results were assessed and graded using the system shown in Table II.

\section{RESULTS}

An analysis of treatment is given in Table III.

Fixation. Fixation was considered to be excellent in 131 cases ( 83 per cent). In two Type B fractures and one Type $\mathrm{C}$ fracture the fixation was only fair because of comminution associated with severe trauma; in such cases bone grafting is now recommended. Fixation was fair in one Type D1 fracture and in 21 Type D2 fractures. The Type D2 fractures had been treated early in the series when we had difficulty in applying the jig and consequently the screws were inserted freehand using a drill guide. With refinements to the instrumentation this problem has now been overcome. In the two Type D2 fractures with poor fixation, faulty placement of the screw was responsible (Fig. 22); retrograde fixation is now preferred for such fractures.

Bone grafting. Cancellous bone grafting was carried out in two acute unstable fractures (Type B) and in nine of the 38 fibrous non-unions (Type D1). Corticocancellous grafting was performed in all Type D2 fractures once it became clear, early in the series, that such fractures would not unite with simple screw fixation alone.

Postoperative immobilisation. A Colles-type plaster was used for an average of four weeks in 15 of the acute unstable fractures, where there had been significant associated damage to ligaments. Initially, plaster was used after bone grafting in the Type D2 fractures, particularly when fixation was only fair. However, with refinements in the technique, plaster is no longer used and there has been a corresponding improvement in the results.

Disability. Of major significance in assessing the results of this technique is the fact that, even with bone grafting, the average time for return to work was between five and seven weeks. Patients who were not entitled to compensation were off work for an average of 9.8 weeks before operation and 5.5 weeks after; the average time for patients claiming compensation was 28 weeks before and
Table III. Analysis of treatment in 158 patients

\begin{tabular}{|lllllll|}
\hline $\begin{array}{l}\text { Type of } \\
\text { fracture }\end{array}$ & Number & \begin{tabular}{l} 
Fixation \\
\cline { 2 - 7 } Excellent
\end{tabular} & Fair & Poor & $\begin{array}{c}\text { Bone } \\
\text { graft }\end{array}$ & $\begin{array}{l}\text { Postoperative } \\
\text { immobilisation* }\end{array}$ \\
\hline Type B & 22 & 20 & 2 & - & 2 & 15 \\
Type C & 21 & 20 & 1 & - & - & - \\
Type D & 38 & 37 & 1 & - & 9 & - \\
Type D2 & 77 & 54 & 21 & 2 & 70 & 28 \\
\hline Total & 158 & 131 & 25 & 2 & 81 & 43 \\
\hline
\end{tabular}

* The average period of immobilisation in a plaster cast was four weeks

6.7 weeks after. Most patients were allowed to swim or play gentle sports within a few weeks of the operation, although violent sports were discouraged until the fracture had united.

Follow-up study. Twelve of the 158 patients were lost to review and eight were eliminated due to insufficient follow-up. The results of 138 patients were therefore

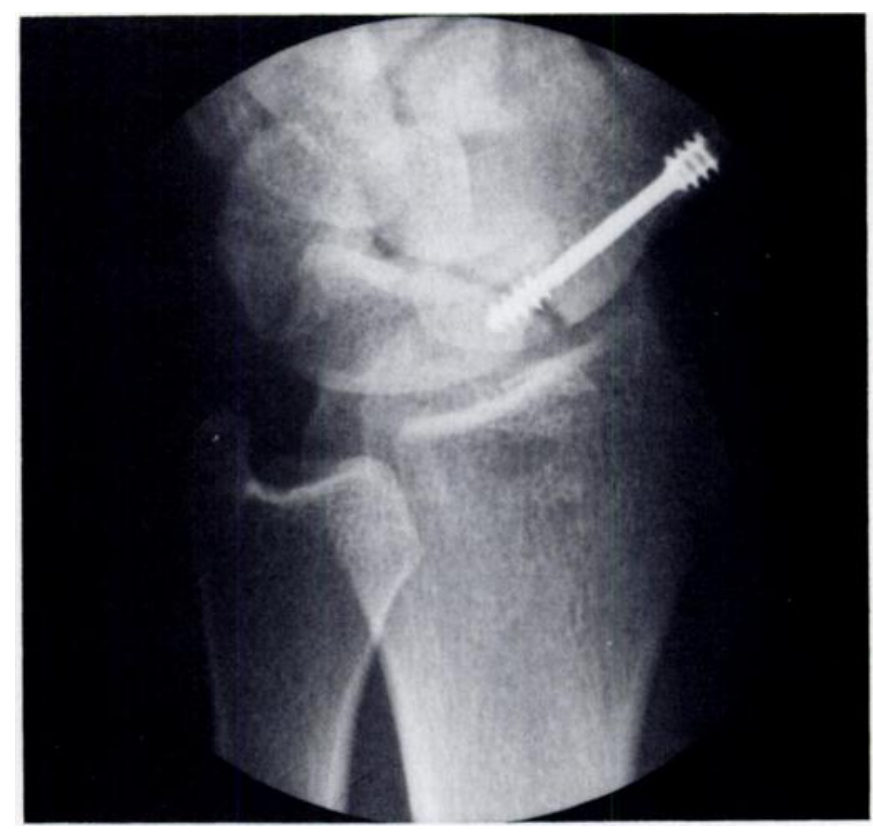

Fig. 22

Radiograph showing faulty screw placement with poor fixation. Retrograde insertion would have been preferable. 
available. A summary of results is given in Table IV. There was a high degree of patient satisfaction (Grades 0 and $1 ; 96$ per cent). The only patient who considered his condition to be worse (Grade 3) was involved in a Workers' Compensation case; his fracture had in fact united. The great majority of patients (91 per cent) had a satisfactory clinical result (Grades 0 and 1), although it was found that stiffness of the wrist, particularly in patients previously treated in plaster, often took a long time to improve. The radiographic result was satisfactory (Grades 0 and 1 ) in 83 per cent of patients, the failures occurring almost exclusively in Type $D$ fractures.

Table IV. Summary of results

\begin{tabular}{|llll|}
\hline Grade & $\begin{array}{l}\text { Patient } \\
\text { satisfaction }\end{array}$ & $\begin{array}{l}\text { Clinical } \\
\text { result }\end{array}$ & $\begin{array}{l}\text { Radiographic } \\
\text { result }\end{array}$ \\
\hline 0 & 106 & 80 & 101 \\
1 & 26 & 45 & 14 \\
2 & 5 & 12 & 11 \\
3 & 1 & 1 & 12 \\
\hline
\end{tabular}

There were no failures of union after operation for Type B fractures (Table V). Two Type $C$ fractures had doubtful union, both after fracture-dislocations without bone grafting; both had satisfactory clinical results. Similarly, in Type D1 fractures, failure to carry out bone grafting appeared to be the prime cause of non-union; one of these fractures required revision. It has now become clear that, even when there is a firm fibrous union, the pseudarthrosis should be excised and grafted before screw fixation to ensure a satisfactory outcome.

Table V. Radiographic results

\begin{tabular}{|lllll|}
\hline \multirow{2}{*}{$\begin{array}{l}\text { Radiographic } \\
\text { assessment }\end{array}$} & \multicolumn{4}{l}{ Fracture type } \\
\cline { 2 - 5 } & Type B & Type C & Type D1 & Type D2 \\
\hline Sound union & 19 & 11 & 29 & 42 \\
Apparent union & 1 & - & 1 & 12 \\
Doubtful union & - & 2 & 2 & 7 \\
Non-union & - & - & 2 & 10 \\
\hline
\end{tabular}

In Type D2 fractures, non-union was associated with a number of factors: the majority of cases were treated over 18 months after injury, and a number of these had already undergone previous operations; 10 were proximal pole fractures with sclerotic bone; and seven fractures (treated early in the series) were not grafted. Five Type D2 fractures required revision.

Complications. There have been no serious complications. One case of superficial wound infection cleared rapidly with antibiotics. Several of the female patients showed a tendency to hypertrophic scar formation, and two patients sustained damage to the palmar cutaneous branch of the median nerve. In three patients there was some degree of proximal penetration of the screw due to faulty technique, causing minor symptoms which were relieved after removal of the screw once the fracture had united.

Revisions. Six patients required revision. Two sustained further severe trauma to the wrist before bony union had occurred and fractured the screw; one of these united after further bone grafting and screw fixation, and the other had a peg-bone graft which failed when the plaster was removed a few weeks after operation. Two cases of non-union after screw fixation but without bone grafting became symptomatic after further trauma; radiographs showed loosening of the screw and both were successfully treated by bone grafting. Silastic replacement was carried out in two patients with non-union who subsequently developed avascular necrosis of the proximal fragment.

\section{DISCUSSION}

The aim of this work was to achieve a simple and yet effective means for producing satisfactory internal fixation of scaphoid fractures, the need for which becomes apparent to anyone who deals with a significant number of these injuries. In spite of regular reports in the literature of a satisfactory outcome after conservative treatment, the true incidence of non-union with conventional methods appears disturbingly high. Similarly the morbidity associated with prolonged use of plaster is seldom acknowledged. Nevertheless, the needs and wishes of the patient should be taken into consideration when planning management; the number of patients seeking treatment with this new technique must be some indication of dissatisfaction with conventional methods.

The results reported in this series appear to justify the use of this new technique. The number of acute fractures treated is relatively small, although the success to date has been most encouraging. Its continued use in the management of unstable acute (Type B) fractures appears to be justified, not only in terms of the rate of union, but also because it avoids prolonged immobilisation. However, it is important to note that unstable comminuted fractures, particularly those associated with dislocations of the midcarpal joint, are technically the most difficult ones to fix. One should not hesitate to carry out primary bone grafting in the presence of comminution and collapse, and it is sometimes advisable to wait for one or two weeks before operating, provided that the dislocation has been reduced.

Screw fixation of stable (Type A) fractures has not been recommended since they appear to do well with only minimal immobilisation in plaster. Nevertheless it may be that economic pressures or the patient's preferences may lead one to consider internal fixation in this group as well. When an acute fracture has been treated 
in plaster, we consider that immobilisation should not be continued for more than six weeks. If, after this time, there is still clinical or radiographic suspicion of persistence of the fracture, then surgery would appear to be the treatment of choice. We cannot agree with London (1961) that such signs can be ignored since non-union, if untreated, will almost inevitably lead to progressive carpal collapse and osteoarthritis.

With symptomatic non-union, our method of treatment offers significant advantages over standard forms of bone grafting: the combination of rigid internal fixation with anterior wedge grafting not only corrects the carpal deformity (Fisk 1970) but also allows early functional recovery of the wrist by avoiding the use of plaster. Internal fixation alone is inadequate for established non-union since the fracture is most unlikely to unite, and loosening of the screw is almost inevitable; this was also apparent in the series reported by Maudsley and Chen (1972). We feel that it is essential to resect the pseudarthrosis and insert a bone graft before screw fixation to give the best chance of bony union. Apart from the small number of technical failures, non-union after this procedure would almost certainly have occurred whatever other type of bone grafting procedure had been performed. In such cases the bone at the fracture site was always sclerotic, thus having a reduced potential for regeneration and bony union. Perhaps consideration might be given to combining this technique with some form of electrical stimulation in these circumstances.

It has been our policy to carry out reconstruction whenever possible, even if it was clear at operation that the chances of union were not high. The fact that a number of such cases did go on to union justifies this approach, and it certainly seems better to preserve the patient's own bone whenever possible, rather than to resort to some alternative procedure such as Silastic replacement, proximal row carpectomy or fusion of the wrist. The low rate of revision would appear to support this approach to the management of such difficult cases. Only when the proximal pole fragment was found to be soft and fragmented was reconstruction not attempted; under these circumstances the proximal fragment was excised and a specially fashioned Silastic prosthesis inserted in its place, stabilised by a stem locked into the distal fragment of the scaphoid. Avascular necrosis of the proximal fragment is often difficult to diagnose radiographically and whenever there is a possibility that reconstruction may not be possible, the patient is warned that the appropriate operative procedure will only become apparent once the fracture has been explored.

Removal of the screw is not normally indicated. Early in the series a number of screws were removed and in all cases there appeared to be no reaction to the implant; indeed it appeared that more damage was done by searching for and removing the screw than would have been the case had it been left in place. Long-term follow-up will show whether the policy of leaving the screw in place is justified.

It is attractive to consider the use of the new bone screw at other sites; its size, ease of insertion, security of fixation and lack of a protrusive head would appear to offer significant advantages over standard implants whenever small cancellous bone fragments need to be fixed. Investigations are currently being carried out using the screw in a number of other sites and early experience has been encouraging.

In conclusion, this technique appears to have overcome the problems previously associated with internal fixation of scaphoid fractures. It offers significant advantages in the overall management both of acute fractures as well as of established non-union. The simple yet effective fixation achieved ensures the best possible outcome while at the same time allowing early functional recovery of the wrist.

We are extremely grateful to those surgical colleagues who supported this project by referring their patients for treatment. We also wish to thank Zimmer USA who funded the development of the instrumentation.

\section{REFERENCES}

Cooney WP, Dobyns JH, Linscheid RL. Fractures of the scaphoid: a rational approach to management. Clin Orthop 1980;149:90-7.

Dooley BJ. Inlay bone grafting for non-union of the scaphoid bone by the anterior approach. J Bone Joint Surg [ Br] 1968;50-B:102-9.

Fisk GR. Carpal instability and the fractured scaphoid. Ann $R$ Coll Surg Engl 1970;46:63-76.

Gasser H. Delayed union and pseudarthrosis of the carpal navicular: treatment by compression-screw osteosynthesis. A preliminary report on twenty fractures. J Bone Joint Surg [ Am] 1965;47-A:249-66.

Herbert TJ. Scaphoid fractures and carpal instability. Proc R Soc Med 1974;67:1080.

Leslie IJ, Dickson RA. The fractured carpal scaphoid: natural history and factors influencing outcome. J Bone Joint Surg [Br] 1981;63-B: 225-30.

London PS. The broken scaphoid bone: the case against pessimism. J Bone Joint Surg [Br] 1961;43-B:237-44.

McLaughlin HL. Fracture of the carpal navicular (scaphoid) bone. J Bone Joint Surg [Am] 1954;36-A : 765-74.

Maudsley RH, Chen SC. Screw fixation in the management of the fractured carpal scaphoid. J Bone Joint Surg [Br] 1972;54-B:432-41

Mulder JD. The results of 100 cases of pseudarthrosis in the scaphoid bone treated by the Matti-Russe operation. J Bone Joint Surg [Br] 1968; 50-B: $110-5$

Russe O. Fracture of the carpal navicular: diagnosis, non-operative treatment, and operative treatment. J Bone Joint Surg [Am] 1960;42-A: 759-68.

Trojan E. Grafting of ununited fractures of the scaphoid. Proc R Soc Med 1974;67: 1078-80. 